\title{
Development and operating environments for a network of Walter/Palya experiment controllers on the Macintosh computer
}

\author{
RON WEISMAN \\ Queen's University, Kingston, Ontario, Canada \\ and \\ WILLIAM L. PALYA \\ Jacksonville State University, Jacksonville, Alabama
}

\begin{abstract}
A program development and an operating environment for using the Walter/Palya experiment controller with a Macintosh are discussed. The importance in maintaining the productivity and intuitive aspects of the Macintosh's user interface is stressed. With this environment, simple choices using the mouse control most aspects of developing and debugging programs and the dayto-day conduct of research.
\end{abstract}

The Walter/Palya experiment controller (Walter \& Palya, 1984) allows researchers to present complex procedures and record the results efficiently. ${ }^{\mathrm{t}}$ It is possible to program and run these single-board computers individually in stand-alone mode from terminals. However, in a busy laboratory, it is more likely that a network of controllers will be connected to a microcomputer via a single serial port. This approach provides the advantages of distributed processing: Networked controllers conduct sessions independently of one another and of the host microcomputer. In my laboratory, a Macintosh Plus is the host computer for a network of 12 experiment controllers.

Laboratories using networked controllers need to provide an efficient and user-friendly software environment on the host for developing and operating ECBASIC programs. In the development environment, an ECBASIC programmer needs a text editor for entering, editing, and storing programs; a communications program for downloading programs and uploading output during debugging; and a few specialized programming tools for line renumbering and variable renaming. In the operating environment, an experimenter wants easy-to-learn, error-free communications procedures for downloading ECBASIC programs to the controllers to run experiments, and for uploading experimental data from the controllers for storage and analysis.

The purpose of this paper is to describe an approach to providing these environments on the Macintosh. My solution uses an existing communications program and text editor. Only the ECBASIC programmer's tools (cur-

This work was supported by an equipment grant from the Natural Sciences and Engineering Research Council of Canada. Address correspondence to Ron Weisman, Department of Psychology, Queen's University, Kingston, ON K7L 3N6, Canada. rently being developed) are written from scratch for this application.

Red Ryder 9.4 was chosen to provide asynchronous serial communication between the Macintosh and the experiment controller network. ${ }^{2}$ Red Ryder has a comprehensive and powerful language with which to specify various communication procedures, the ability to specify a series of procedures with a simple macro command, and the ability to easily send the control characters required to implement a true network. All of these characteristics are necessary if a coherent environment for running experiment controllers is to be developed. Notepad+ was chosen for composing ECBASIC programs and Red Ryder procedures. ${ }^{3}$ Notepad + is a simple, intuitive fullscreen editor.

The great power of the Macintosh comes from its ability to provide an environment that enhances productivity as well as its being user friendly and easy to learn. The advantages of the Macintosh environment cannot be overstated. A major factor in the productivity enhancement made possible by a Macintosh is that it provides a desktop workspace and allows concurrency among "desk accessory" programs. A click of the mouse allows a user to quickly and conveniently switch between alternating tasks, such as between writing and testing experiment control programs or between downloading to and uploading from controllers. Without the concurrency provided by Macintosh's desk accessories, the programmer is faced with the time-consuming process of alternately quitting and opening various utilities. The text editor and programming tools function as desk accessories to the communications program.

\section{Program Development Environment}

Program development involves entering, editing, and saving an experiment control program using Notepad+. 
The entire program, or a subset, is then downloaded to a controller for testing by using Red Ryder. As ECBASIC programs are written and saved, loaded and tested, and then modified and retested, a programmer can switch between the tasks with simple clicks of the mouse. These working programs can subsequently be used for running experiments on the controllers in the operating environment.

Red Ryder will automatically carry out a variety of procedures. They are set up with the procedure file illustrated in Listing 1. Subsequently, the start-up procedure, "EC Environment," initializes Red Ryder for communication with the experiment controllers and sets up the program development environment, when chosen. As shown in Listing 1, the start-up procedure provides, among other attributes, half duplex communication at 9600 baud, no parity, 8 data bits, 1 stop bit, and a Teletype-like 80-character dumb terminal format. ${ }^{4}$

Red Ryder provides "virtual buttons" that are displayed 10 at a time in a macro status bar across the top of the screen. Each button, when clicked, sends a command of up to 40 characters. We have constructed a variety of communication procedures to create an ECBASIC program development environment. The user need only point to the name of the desired sequence and click the mouse.

We use Box 1 for all program development and testing. The first two macros on the macro bar bring Experiment Controller 1 on-line and take it off-line. This allows us to easily establish or break a direct link to Box 1 . Red Ryder sends $<$ control $\mathrm{A}\rangle\langle 1\rangle<$ shift $\mathrm{S}\rangle$ when the macro BOX ON is clicked and $\langle$ control $S>$ when the macro BOX OFF is clicked. The macro TEST PROGRAM brings Box 1 on-line and downloads the current file under development, called "PROGRAM UNDER TEST," into Box 1, whereas the macro TEST DATA brings Box 1 on-line, uploads data from Box 1, and saves

\section{LISTING 1}

EC Environment Procedure

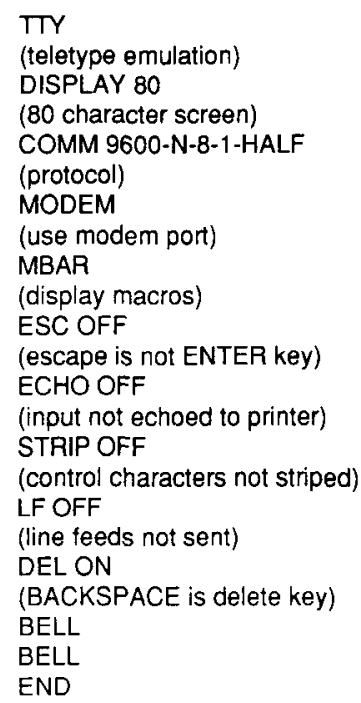

LISTING 2

Test Program Procedure

TYPE ^A1 $\mathrm{S}^{\wedge} \mathrm{M}$

(wake up box 1)

PAUSE

(pause 2 see)

TYPE ${ }^{\wedge} \mathrm{C}^{\wedge} \mathrm{M}$

(reset experiment controller)

PAUSE

TYPE NEW ^ $M$

(clear box 1 program buffer)

PAUSE

SENDA RED RYDER:EC STUFF:PROGRAM UNDER TEST

(send file "program under test")

BELL

BELL

END

LISTING 3

Test Data Procedure

RECA RED RYDER:EC STUFF:RECEIVED TEST DATA

(open data file "receive test data")

TYPE ^A1 ${ }^{\wedge} M$

(permission to box 1 to upload data)

PROMPT*END OF DATA*

(collect data till"*end of data*"

TYPE ^ $S^{\wedge} M$

(disconnect box 1 from network)

CLOSE

(end receive and close file)

BELL

BELL

END

it in the standard upload test file "RECEIVED TEST DATA." The KILL macro terminates the execution of the program under development running in Box 1.

The TEST PROGRAM macro, shown in Listing 2, first sends the wake-up code for Controller 1 , followed with a carriage return (TYPE ${ }^{\wedge} \mathrm{A} 1 \mathrm{~S}^{\wedge} \mathrm{M}$ ). It then pauses, sends a $<$ control $\mathrm{C}>$ carriage return to reset the controller, pauses, clears the program buffer (TYPE NEW ${ }^{~} \mathrm{M}$ ), and then sends the file containing the program under development, "PROGRAM UNDER TEST," which it gets from the EC STUFF folder on the Red Ryder disk. The program in "PROGRAM UNDER TEST" is expected to have OFFRUN as its last instruction (see Figure 3). As a result, after the experiment control program has been loaded by the controller, the controller disconnects from, or releases, the network and begins executing that program.

The TEST DATA macro, shown in Listing 3, opens the standard upload test file "RECEIVED TEST DATA" on the disk, and then gives Box 1 permission to upload data. Networking is accomplished in the Walter/Palya experiment controllers by halting program execution if a PRINT statement is encountered when the box does not have permission to use the network line. (In the example program in Figure 3, program execution will halt at line 20.) $<$ Control $\mathrm{A}><1><$ shift $\mathrm{S}>$ gives that permission to Box 1. Box 1 then proceeds to execute its PRINT statements. When all data have been transmitted, the controller is expected to send *END OF DATA* 
(line 130 in Figure 3), which is used by the upload program to cause the file to be closed. The TEST DATA macro then sends < control $\mathrm{S}>$, which directs any box on the line to disconnect or release the network line.

A typical program development session is initiated by inserting the Red Ryder disk and by double clicking the EC ENVIRONMENT icon. This loads and properly configures Red Ryder. The user then switches to Notepad+, creates a blank page, and writes a program (see Figure 1). The program is saved by using the Notepad+ pull-down menu (see Figure 2). This test program is then downloaded to the experiment controller by clicking the macro TEST PROGRAM (see Figure 3). If changes in the control program are desired, the program is recovered simply by clicking Notepad + . The code to be altered is selected (see Figure 4) and changed, and the revised program is then saved. The updated file is then sent to the controller again by clicking the macro TEST PROGRAM. When finalized, the experiment control program is stored with Notepad+ under its desired name (see Figure 5).

\section{Operating Environment}

An ideal operating environment would provide a Macintosh user interface to make running experiments easy to

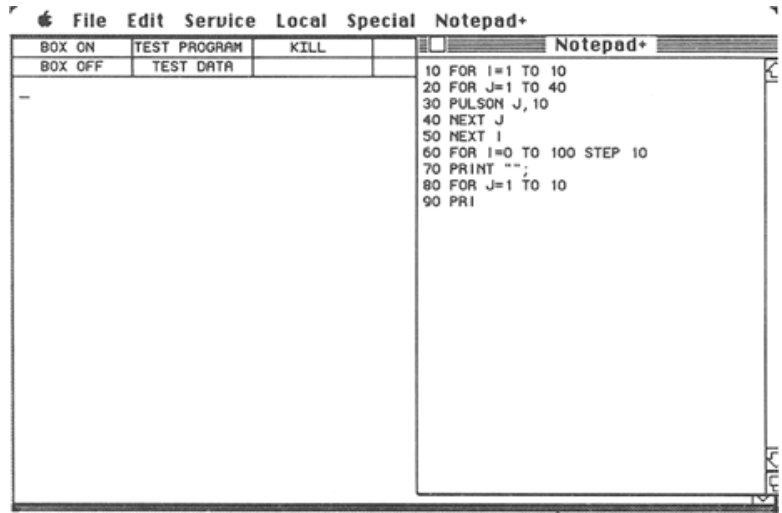

Figure 1. A Macintosh screen that illustrates an ECBASIC program being written with Notepad+.

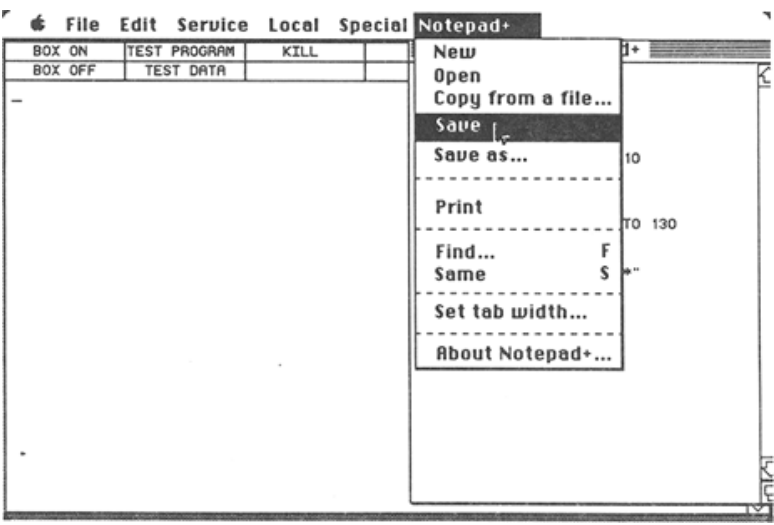

Figure 2. A Macintosh screen that illustrates saving a program with Notepad+'s pull-down menu.

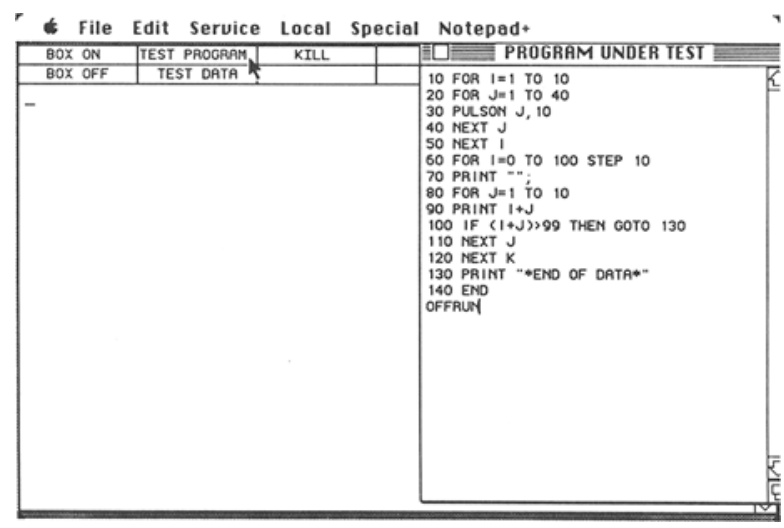

Figure 3. A Macintosh screen that illustrates the operation of the Red Ryder macro, which will send the program under development to Experiment Controller 1.

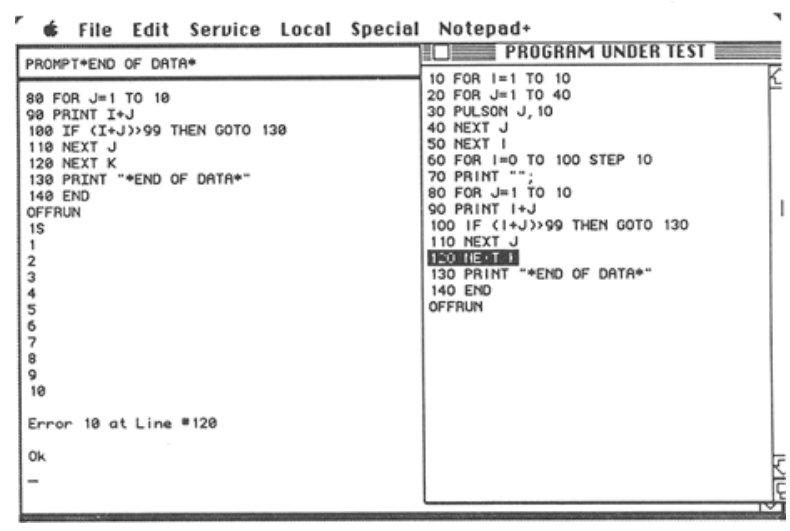

Figure 4. A Macintosh screen that illustrates the selection of a program line where corrections must be added.

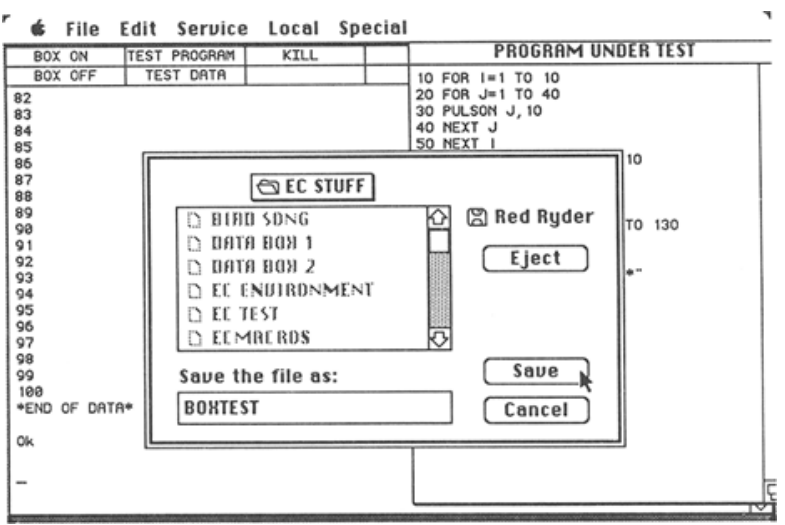

Figure 5. A Macintosh screen that illustrates saving a program that is under development under its final name.

learn and error free. Research assistants should be able to load experiment controllers en masse or individually, using only the mouse. Similarly, uploading and saving data to the Macintosh should be accomplished with the mouse. By avoiding the keyboard, one can avoid typing errors and confusing command codes. Again, Red Ryder's macro buttons can be used for this purpose. The upload- 
ing or downloading of the appropriate files can be carried out automatically by Red Ryder as the result of single clicks. Example Red Ryder communication sequences for loading programs (TEST PROGRAM) and saving data (TEST DATA) are shown in Listings 2 and 3, respectively.

The operating environment macros are designed to simplify the process of carrying out day-to-day procedures in a research laboratory. Macros can be designed to implement procedures specifically tailored to the needs of each lab. Macros can upload data and maintain separate files for each, or they can append consecutive sessions to the same file. Manually entered identifiers, such as dates, times, and subject numbers, can be appended to files before they are stored. Macros can load individual boxes with individual experiment control programs, or macros can be designed to run a squad with the same procedure in all boxes at once. Utility macros such as KILL ALL BOXES are also very useful.

It is important to plan ahead when writing the data output portion of experiment control programs (i.e., PRINT commands). Data files should conveniently load into a powerful spreadsheet, such as Microsoft Excel, for manipulation and analysis. Macintosh spreadsheets require tabs between columns and carriage returns between rows. The sample program (PROGRAM UNDER TEST), shown in Figure 1, illustrates the placement of tabs ("control I") between consecutive datum output by the PRINT statement. Uploaded files retain these tabs. ${ }^{5}$ The resulting processed data files can then be opened with Excel.

The procedures described here have been tested with a network of experiment controllers and a Macintosh Plus. After acquiring more skill with Red Ryder and ECBASIC, one might develop more elaborate procedures and, perhaps, seek further programming tools, such as a syntax checker and an experiment controller simulator.
However, the current procedures should allow investigators to get on with their experiments with a minimum of additional software development.

\section{REFERENCES}

Walter, D. E., \& Palya, W. L. (1984). An inexpensive experiment controller for stand-alone applications or distributed processing networks. Behavior, Research Methods, Instruments, \& Computers, 16, 125-134.

WALter, D. E., \& PAlya, W. L. (1985). Document set for experiment controller. Jacksonville, AL: Jacksonville State University, Department of Psychology.

\section{NOTES}

1. Detailed information about the experiment controller is available in Walter \& Palya's (1985) Document Set for Experiment Controllers, which is available from W. L. Palya, Department of Psychology, Jacksonville State University, Jacksonville, AL 36265.

2. Red Ryder 9.4 is shareware available on various electronic bulletin boards. Red Ryder has been updated to version 10, a commercial product of The Freesoft Co., 150 Hickory Dr., Beaver Fall, PA 15010. The procedures and macros used in communicating with the Walter/Palya experiment controllers were developed with version 9.4, but can be modified to operate with version 10 by resaving them as text in version 9.4 and then compiling in version 10 .

3. Notepad+ is a component of SideKick 2.0, a product of Borland International, 4585 Scotts Valley Dr., Scotts Valley, CA 95066. Any text editor available as a desk accessory and capable of saving text-only files will serve.

4. Half duplex displays the file transmissions to the experiment controller on the screen, whereas full duplex does not. The start-up procedure also specifies that Red Ryder is to wait for a line feed before transmitting the next line of the experiment control program. In this way, the network provides for limited error checking and hand shaking.

5. Unfortunately, other control characters are sometimes added in this process. The latter can be removed with Tab Expander, a shareware desk accessory. It is a product of SysCon Consulting, 9001 Laurel Dr., Austin, TX 78758. Processed files should be given a special extension denoting that they have been processed (e.g., .SS) to avoid confusion. 